\title{
VERY HIGH-FIELD SHORT-PULSE DIPOLE MAGNET FOR COMPACT PROTON SYNCHROTRON
}

\author{
S. Tokura, A. Hirata, K. Shouji, S. Nakajima, Y. Miyauchi, A. Noda*, Y. Iwashita*, T. Shirai*, \\ and K. Endo** \\ Ishikawajima-Harima Heavy Industries Co. Ltd., Yokohama 235-8501, Japan \\ *Institute for Chemical Research, Kyoto University, Uji, Kyoto 611-0011, Japan \\ **High Energy Accelerator Research Organization (KEK), Oho 1-1, Tsukuba 305-0801, Japan
}

\section{Abstract}

Design of a short-pulse dipole magnet for a compact proton synchrotron dedicated for cancer therapy is presented. Its pulse width and maximum magnetic field are 7 [ms] and 4 [T], respectively. Sextupole coefficient is reduced to $4 \times 10^{-4}\left(32\left[\mathrm{~T} / \mathrm{m}^{2}\right]\right)$ at $4[\mathrm{~T}]$ by the optimization of the magnet geometry, which is estimated to be sufficiently small for beam acceleration. Laminated silicon steel ( thickness of 1 [mm]) is enough to suppress the eddy current effect in the yoke and B-constant shape rather than Rogowski's curve is adopted at the end of the magnet.

\section{INTRODUCTION}

For wide spread use of proton radiation therapy, which is mild for patients suffering from cancer, proton synchrotrons are required to be decreased in size of total system or to be reduced in its cost. To attain a sufficient energy for cancer therapy in a very compact scale, the magnetic field of the normal-conductive bending magnet have to be excited up to $3-5$ [T]. Proton synchrotron using pulsed high field magnet $(5[\mathrm{~T}])$ was proposed by BINP [1], and component development had been made [2]. However, field distortion (especially sextupole component) of dipole magnets remained to be improved. Pecardi et al.[3] proposed the magnet with magnetic field of 4 [T] (referred as STAC magnet hereafter). Recently the design of dipole magnet with magnetic field of 3 [T] was proposed by one of the authors (K. Endo, KEK) [4], and good possibility to realize a very small synchrotron was suggested. Starting from parameters of STAC magnet, we tried to design very high-field, short pulse dipole magnet for compact proton synchrotron dedicated for medical use.

\section{MAIN PARAMETERS OF THE SYNCHROTRON AND ITS DIPOLE MAGNET}

A compact $200[\mathrm{MeV}]$ proton synchrotron with the injection energy of $1[\mathrm{MeV}]$ has been developed. Four bending magnets and two quadrupole magnets form the basic lattice. As the outer dimension is about 2.3 [m], it can be transported to hospitals after the assembly and tuning in the factory. Main parameters of synchrotron and its dipole magnet are listed in Table 1.

Table 1 Main parameters of the synchrotron and its dipole magnet

\begin{tabular}{|l|l|}
\hline Item & Value \\
\hline Maximum energy & $200[\mathrm{MeV}]$ \\
\hline Injection energy & $1[\mathrm{MeV}]$ \\
\hline Circumferences & $6.393[\mathrm{~m}]$ \\
\hline Average radius & $1.017[\mathrm{~m}]$ \\
\hline Tune, $v_{\mathrm{x}} / \mathrm{v}_{\mathrm{y}}$ & $1.41 / 0.45$ \\
\hline Rf frequency & $2.16-26.5[\mathrm{MHz}]$ \\
\hline Accumulated protons & $1.5 \times 10^{9}$ \\
\hline Bending radius & $0.54[\mathrm{~m}]$ \\
\hline Bending angle & $90[\mathrm{deg}]$ \\
\hline Max. bending field & $4[\mathrm{~T}]$ \\
\hline Max. current & $200[\mathrm{kA}]$ \\
\hline Acceleration & $3.5[\mathrm{~ms}]$ \\
\hline Inductance & $0.4[\mathrm{mH}]$ \\
\hline Voltage & $40[\mathrm{~V}]$ \\
\hline Number of turn & $1[$ turn/coil] $]$ \\
\hline Gap height/width & $0.05 / 0.02[\mathrm{~m}]$ \\
\hline (Major parameters follow STAC design )
\end{tabular}

(Major parameters follow STAC design )

\section{DIPOLE MAGNET DESIGN}

\subsection{Reduction of multipole coefficients}

Figure 1 shows the shape of the dipole magnet. Magnetic fields were analysed using a 3 dimensional, time dependent code "JMAG"[5]. The excited current pattern of the dipole magnet is a quarter wave length of a sine curve. From symmetries of the ideal magnet, only quadrant was analysed.
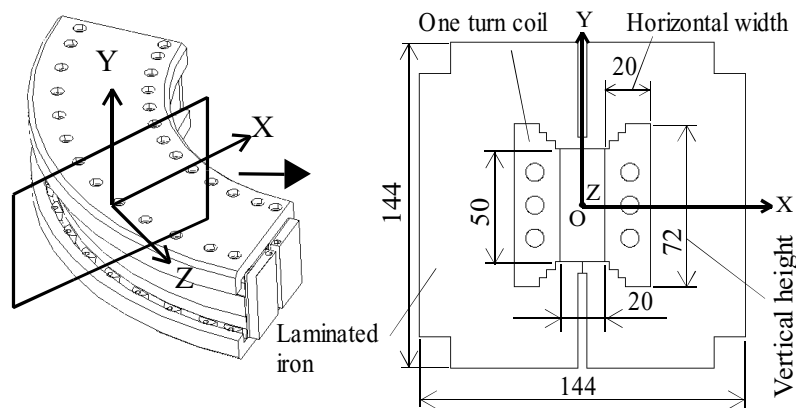

Figure 1:Shape of the dipole magnet (top) and its cross-section (bottom) 
Magnetic field in the dipole magnet is expanded as follows.

$$
\mathrm{B}_{\mathrm{y}}=\mathrm{B}_{0} \Sigma_{\mathrm{n}=0}\left(\mathrm{r} / \mathrm{r}_{0}\right)^{\mathrm{n}}\left\{\mathrm{a}_{\mathrm{n}} \cos (\mathrm{n} \theta)+\mathrm{b}_{\mathrm{n}} \sin (\mathrm{n} \theta)\right\}
$$

Where $\mathrm{B}_{\mathrm{y}}$ is $\mathrm{y}$ component of the field, and $\mathrm{B}_{0}$ the field at the center of the magnet. The $a_{n}$ and $b_{n}$ are normal and skew 2(n+1)-pole coefficients, and $r_{0}$ is a radius of reference circle (In this study, $\mathrm{r}_{0}$ is $10[\mathrm{~mm}]$ ). Considering the symmetry of the magnet, $b_{n}$ is always zero if we neglect fabricated error. In generally, sextupole component can be expressed as follows.

$$
\frac{1}{B_{0} \cdot \rho} \cdot \frac{\partial^{2} B}{\partial x^{2}}
$$

Where, $\rho$ is bending radius of dipole magnet. For example, sextupole component on $\mathrm{X}$ axis can be expressed by $2 \mathrm{a}_{2} /\left(\rho \mathrm{r}_{0}^{2}\right)$.

In order to keep enough size of dynamic aperture during acceleration, the sextupole coefficient $\left(\mathrm{a}_{2}\right)$ must be controlled at least under $2 \times 10^{-3}$ [3].

We studied the effect of the coil geometry on the field distortion. As shown in Figure 2, peak value (mostly at $3.5[\mathrm{~ms}]$ ) of $\mathrm{a}_{2}$ was much influenced by vertical height of the coil. On the contrary, reducing horizontal width of coil to $6[\mathrm{~mm}]$ (which is almost skin depth of the coil), $\mathrm{a}_{2}$ wasn't influenced so much (not showing in the figure). Figure 3 shows the typical J-MAG outputs representing the contour of the current density distribution in the coil with rectangular shape at different time. Due to current penetration into the coil, the distribution of current density changes during acceleration. Considering the penetration into the coil, we optimized the coil geometry by following method. Figure 4 a shows coil geometry used on several design steps, and $4 \mathrm{~b}$ shows time evolution of $\mathrm{a}_{2}$ on each coil geomerty. In the 1-st step, the coil geometry (vertical height: 50 [mm], Figure 4a case1) was chosen, because of small value of $a_{2}$ till 1 [ms] (Figure 2). Saturation of magnetic pole caused remarkable increase on value of $\mathrm{a}_{2}$ after $1[\mathrm{~ms}]$. In the 2-nd step, to decrease $\mathrm{a}_{2}$ after 1 [ms], vertical height of the coil cross-section where current hardly penetrate (Figure 3a, x1-x4) was increased (Figure4a case2). Due to current penetration after $1[\mathrm{~ms}]$, the current density distribution after $1.0[\mathrm{~ms}]$ was changed. As a result, the value of $a_{2}$ after 1 [ms] was decreased (Figure $4 \mathrm{~b}$, case2). In the 3-rd and the 4-th step, vertical height was increased according to current penetration (Figure 4a case 3 and case4), so that optimal geometry with the sextupole coefficient of $4 \times 10^{-4}$ was obtained. As shown in Figure 5, the size of dynamic aperture which was calculated using the optimal value of $\mathrm{a}_{2}$ and $\mathrm{a}_{4}$ (case4) is kept during acceleration. Detailed consideration about lattice design or dynamic aperture were reported in the previous report [6].

The results above shows that eddy current in the coil greatly affects the field distribution. Though we cannot demonstrate here for lack of space, this is even more

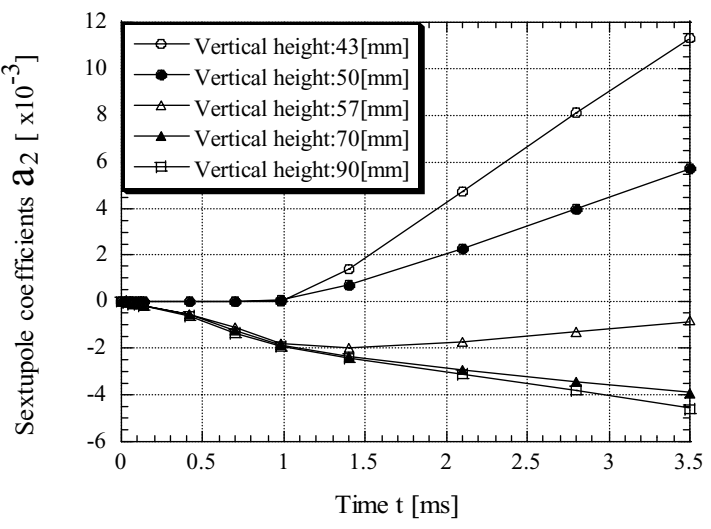

Figure 2:Relation between vertical height of coil geometry and time evolution of sextupole coefficient

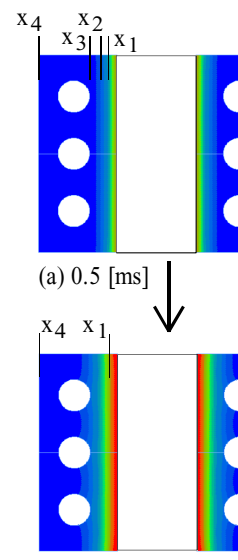

(b) 1.0 [ms]
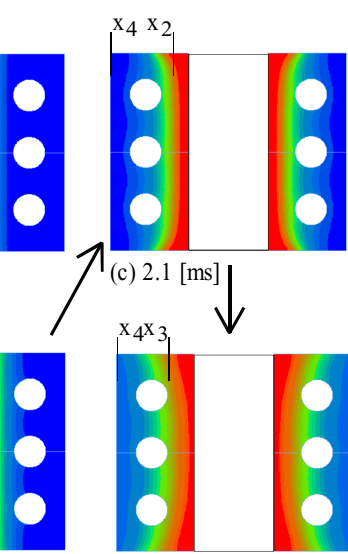

(d) $3.5[\mathrm{~ms}]$

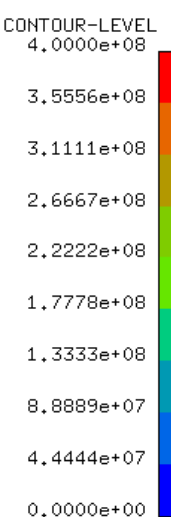

[A/m2]
Figure 3: Current density distribution contour in the coil at different time

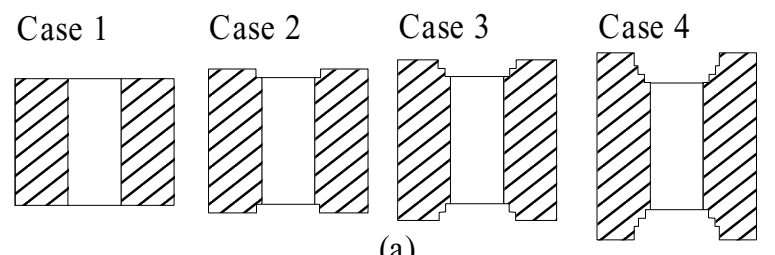

(a)

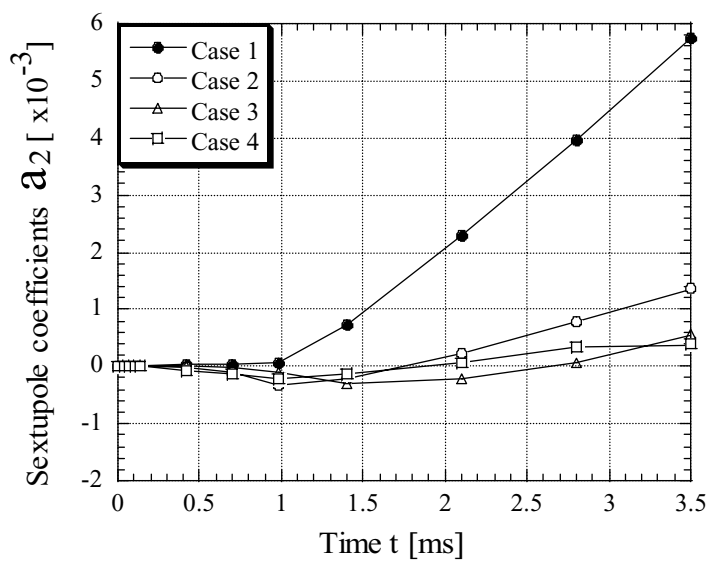

(b)

Figure 4: Coil geometry on several design steps (a), and time evolution of sextupole coefficient on each coil geometry (b). 


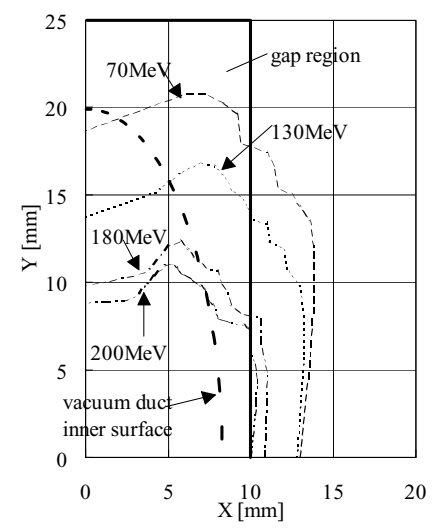

Figure 5:Energy evolution of dynamic aperture obvious comparing those results (Figure 2 or 3 ) with those of static calculations or those of time dependent calculations under the condition without eddy current in the coil (correspond to strand coils).

\subsection{Influence of laminated magnetic core}

Using laminated silicon steels, no eddy current was assumed in the computational analysis. Figure 6 shows time evolution of $\mathrm{a}_{2}$ using three types of yoke given as follows. The "ideal" is the yoke in which there is no eddy current, the "not laminated" is the yoke using silicon steel which is not laminated, and the "laminated" is the yoke using laminated silicon steel (silicon steel and its insulator are $1[\mathrm{~mm}]$ and $50[\mu \mathrm{m}]$ in thickness respectively). As shown in the figure, there is almost no difference in the field distortion nor current between the case of "ideal" and "laminated", while those value in the case of "non laminated" are much larger in all range of the field strength.

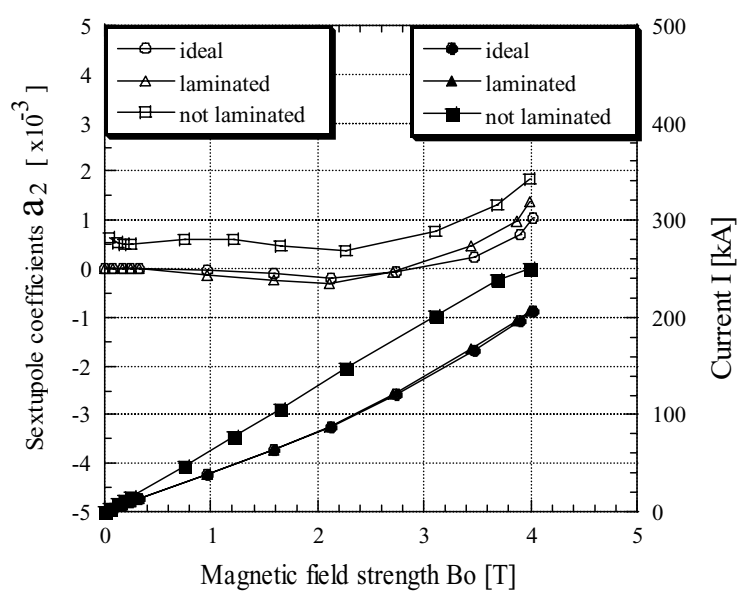

Figure 6:Effect of laminated magnetic core on field distortion

\subsection{Effective length}

Figure 7 shows the time variation of the effective length at the center of the aperture for different longitudinal edge shapes. Before improving the magnet end (rectangular

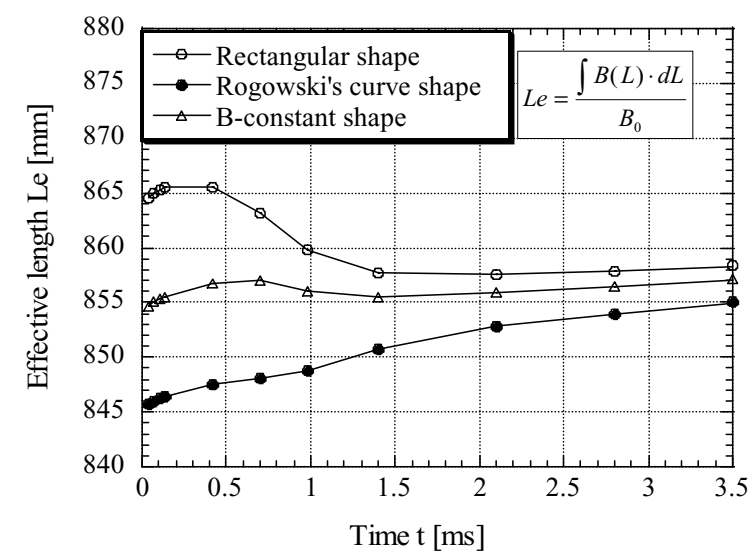

Figure 7:Time variation of the effective length at the center of the aperture shape), there is considerable change of the effective length until $1[\mathrm{~ms}]$, while there is only a slight increase after $1[\mathrm{~ms}]$. In case of the Rogowski curve end, the time variation does not become small. On the other hand, as the magnet ends are cut stepwise along the constant magnetic flux density contour (B-constant shape), the longitudinal edge shape gives smaller time variation of the effective length.

\section{CONCLUSIONS}

Very high-field, short pulse dipole magnet was designed.

To reduce multipole field components, the step shaped coil was designed considering the time evolution of the current distribution in the coil. Using this coil geometry, the sextupole coefficient can be controlled within $4 \times 10^{-4}$ $\left(32\left[\mathrm{~T} / \mathrm{m}^{2}\right]\right.$ at $\left.4[\mathrm{~T}]\right)$, so that enough dynamic aperture can be kept during acceleration. Using the laminated silicon steel (thickness of under $1[\mathrm{~mm}]$ ), eddy current hardly occur in the yoke. Besides, to diminish the time variation of the effective length, the shape of magnet-ends in beam direction should be shaped along the constant magnetic flux density contour. Smaller time variation of the effective length can be obtained.

\section{REFERENCES}

[1] I. Averbukh et al., "Project of Small-Dimensional 200 MeV Protonsynchrotron", EPAC'88, Rome, 1988, pp413-6.

[2] "Prototype of the Proton Synchrotron with 5-Tesla Dipole Magnets", BINP annual report, 1998, pp207-9.

[3] L. Picardi et al., "Preliminary Design of a Technologically Advanced and Compact Synchrotron for Protontherapy", ENEA, 1994.

[4] K. Endo et al., "Table-top Proton Synchrotron Ring for Medical Applications", EPAC2000, Vienna, 2000, pp2515-7.

[5] "JMAG-Works User's Manual Version 6.0", The Japan Research Institute LTD. Update, 2000.

[6] Y. Miyauchi et al., "Preliminary Study of the Feasibility of Pulse High Field Compact Synchrotron for Medical Use", Kyoto Univ. activity report, Vol.6, 2001. 